that; in the mouse, (1) morphine dependence increases the turnover rate of 5-hydroxytryptamine in the brain and (2) para-chlorophenylalanine, which lowers the brain level of 5-hydroxytryptamine, retards the development of dependence. Mr I. Marshall (St Mary's Hospital Medical School, London) also described his failure independently to confirm these two conclusions of Way and his colleagues.

\section{PHOTOSYNTHESIS}

\section{Chl $b$ Redundant?}

by our Plant Physiology Correspondent

THE final nails seem to have been driven into the coffin of the idea that chlorophyll $b$ plays a fundamental part in the energy trapping reactions of photosynthesis. This molecule, which at one time seemed to be an integral part of the " $z$ " schemethe series of interlinked electron movements powered by light which constitute the primary reactions of photosynthesishas now been relegated to the status of an accessory "spectrum broadening" pigment.

The evidence on which this conclusion is based has been gathered from a number of diverse types of experiments. Chlorophyll $b$ has long been implicated in the photosynthesis of green plants and algae on the basis of action spectra measured for photosynthetic enhancement, and it seemed likely that the pigment was an essential component of photosystem 2, the reaction driven by short wavelength light $(<700 \mathrm{~nm})$ which gives rise to free electrons and the evolution of gaseous oxygen. The first crack in this argument came with the demonstration that the pigment complexes for both photosystem 2 and for photosystem 1 -the reaction driven by $700 \mathrm{~nm}$ light which is ultimately resolved in the production of a source of reducing power-contain a mixture of chlorophyll $a$ and chlorophyll $b$. In photosystem 2 , the ratio of chlorophyll $a$ to chlorophyll $b$ is about 2 , and the corresponding value for photosystem 1 is approximately 6 . Other studies have shown that chlorophyll $b$ does not seem to be necessary for oxygen evolution. H. R. Highkin and A. W. Frenkel experimented with a mutant variety of barley which lacked chlorophyll $b$, and showed that in comparison with the wild type there was no decrease in photosynthetic efficiency or evolution of oxygen (Plant Physiol., 37, 814; 1962).

In blue-green algae it is quite easy to alter the amounts of the various photosynthetic pigments present in the cell by altering the conditions of illumination. B. J. Reger and R. W. Krauss of the University of Maryland have managed to alter the ratio of chlorophyll $a$ to chlorophyll $b$ in cells of the green algae, Chlorella, using this technique (Plant Physiol., 46, 568; 1970). Cells grown at
6,000 foot candles (high light cells) contained, on a dry weight basis, only one tenth the amount of chlorophyll found in cells grown at 300 foot candles (low light cells). Nevertheless, the high light cells had a greater photosynthetic capacity than the low light cells, a rather strange effect which Reger and Krauss attribute either to a larger number of smaller photosynthetic reaction centres in the high light cells or, alternatively, a small number of normal sized but enzymatically more efficient units.

The normal chlorophyll $a$ to chlorophyll $b$ ratio for Chlorella is about 2 . In the high light cells, however, this ratio was increased by the light treatment to 6.2 .

In spite of this apparent deficiency in chlorophyll $b$ in the photosynthetic system, however, the overall photosynthetic product in the high light culture and the low light culture was not greatly different. Furthermore, some photosynthetic enhancement, which could be attributed to the presence of chlorophyll $b$, could be observed even in the high light cultures.
Reger and Krauss suggest that the level of chlorophyll $b$ may reflect a regulatory device which influences the cell's phosphorylation pathways. Their conclusion is based on respiratory data; the rate of respiration in the high light cells was several times greater than that of the low light cells. They suggest therefore that the level of ATP-required for the carbon fixing activities of photosynthesis -may be low in cells where the content of chlorophyll $b$ is small, and that this energy must therefore be provided from respiration. They have circumstantial evidence for this; low light cells were bleached and killed by peroxyoacetyl nitrate, an inhibitor which specifically blocks the reactions by which ATP is built up photosynthetically in the cells. High light cells, on the other hand, were little affected, suggesting they were deriving their energy from systems other than photosynthesis. Whether this suggestion for the role for chlorophyll $b$ is correct or not, it now seems certain that it has little to do with the primary energy trapping reactions.

\title{
A Witches' Brew of Neurotoxins
}

IN next Wednesday's Nature New Biology, Narahashi, Albuquerque and Deguchi describe the effects of batrachotoxin on the membrane of the perfused squid giant axon. Batrachotoxin, an alkaloid isolated from the skin secretion of the frog Phyllobates aurotaenia, is the latest in a series of naturally occurring toxins which have been found to possess unique actions on nervous tissues. The compound was first reported to block neuromuscular transmission in the rat and toad by Märki and Witkop (Experientia, 19, 329; 1963). Narahashi et al. have now recorded the effects of the toxin on the intracellularly recorded membrane potential of the squid axon. Application of batrachotoxin $(1 \mu \mathrm{m})$ to either the outside or the inside of the nerve membrane resulted in a slow and irreversible depolarization of the resting membrane potential. This depolarization was antagonized by the application of tetrodotoxin, which is known to act by reducing the permeability of the nerve membrane to sodium ions (Narahashi et al., Amer. J. Physiol., 198, 934; 1960). Batrachotoxin also failed to cause a depolarization if sodium ions were absent from the external solution. Narahashi et al. conclude that batrachotoxin acts by causing a selective increase in the resting membrane permeability to sodium ions. In spite of this increase in sodium permeability, a further large increase in sodium conductance could still occur when the membrane was electrically depolarized, indicating that the sodium channels which are available for permeability increase on nerve stimulation remain largely unchanged by batrachotoxin.

Tetrodotoxin, the puffer-fish poison, has already proved its worth as a research tool because of its unique effect in blocking the increase in sodium permeability of excitable membranes normally evoked by stimulation. Batrachotoxin, whose effects seem to be almost the converse of those of tetrodotoxin, also seems likely to be a useful new research tool. These findings may tend to confirm the suspicions of those who feel that research in neurobiology seems to involve magic and witchcraft. Drugs from exotic sources certainly play an important part in this field. For example, the snake venom peptide $\alpha$-bungarotoxin (Lee et al., Nature, 215, 1177; 1967) seems likely to be important for studies of cholinergic receptors (Changeux et al., Proc. US Nat. Acad. Sci., 67, 1241; 1970)-supplanting in this role even the more familiar curare, the South American Indian arrow poison. The toxic plant alkaloid bicuculline has also attracted attention because of the report that it represents the first known antagonist of GABA receptors in the nervous system (Curtis et al., Nature, 226, 1222; 1970). If nothing else, these remarkable compounds suggest that nature has so far proved rather more successful than human chemists in devising agents with highly selective actions on the nervous system. 